\title{
SRGN Promotes Colorectal Cancer Metastasis as a Critical Downstream Target of HIF-1 $\alpha$
}

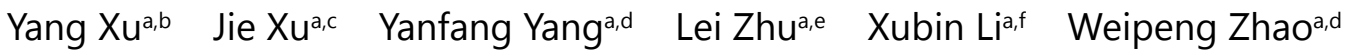

aNational Clinical Research Center of Cancer, Tianjin Key Laboratory of Cancer Prevention and Therapy, Tianjin's Clinical Research Center for Cancer, Tianjin, ${ }^{\mathrm{b}}$ The Second Hospital of Tianjin Medical University, Colorectal Surgery Department, Tianjin, 'Tianjin Medical University Cancer Institute and Hospital, Department of Senior Ward, Tianjin, dDepartment of Breast Cancer, Tianjin Medical University Cancer Institute and Hospital, Tianjin, eDepartment of Molecular Imaging and Nuclear Medicine, Tianjin Medical University Cancer Institute and Hospital, Tianjin, fDepartment of Radiology, Tianjin Medical University Cancer Institute and Hospital, Tianjin, China

\section{Key Words}

Srgn • HIF1 $\alpha \cdot$ Colorectal cancer • Metastasis • Invasion • EMT

\begin{abstract}
Background/Aims: The chondroitin sulfate proteoglycan serglycin (SRGN), a hematopoietic cell granule proteoglycan, has been implicated in promoting tumor metastasis; however, the underlying mechanisms remain to be elucidated. The present study aimed to investigate the SRGN gene expression and its regulation as downstream signaling of hypoxia-inducible transcription factor 1 alpha (HIF-1 $\alpha$ ) in colorectal cancer (CRC) cells and tissues. Methods: The expression of SRGN was analyzed in CRC specimens for its correlation with progression and metastasis. Using chromatin-immunoprecipitation (ChIP), quantitative real-time PCR, Western blot, and transwell assay, the functional role and underlying mechanism of SRGN in CRC metastasis were elucidated. Thus, this study provides evidence of a critical role of SRGN in metastatic progression of CRC. Results: Our results indicated that SRGN overexpression was significantly associated with poor prognosis in CRC specimens. SRGN overexpression promoted CRC cell migration and invasion in vitro; however, $S R G N$ depletion exhibited contrasting effects. Mechanistic investigations revealed that HIF-1 $\alpha$ regulated SRGN transcription via physically binding to a hypoxia response element in its promoter region. Conclusions: In conclusion, we demonstrated that dysregulated HIF-1 $\alpha /$ SRGN signaling promotes CRC progression and metastasis. SRGN may serve as a potential candidate therapeutic target for metastatic CRC.
\end{abstract}

Y. Xu and J. Xu contributed equally to this work.

Jie $\mathrm{Xu}, \mathrm{MD}$

and Weipeng Zhao, MD, PhD
Tianjin Med. Univ. Cancer Inst. and Hospital, National Clinical Research Center of Cancer

Huanhuxi Road, Hexi District, Tianjin, (China)

Tel. +86-022-23340123, E-Mail xujie508@126.com; weipengzhao_oncology@yahoo.com 


\section{Cellular Physiology Cell Physiol Biochem 2018;48:2429-2440 \begin{tabular}{l|l|l} 
and BiOChemistry & $\begin{array}{l}\text { DOI: 10.1159/000492657 } \\
\text { Published online: 15 August, } 2018\end{array}$ & $\begin{array}{l}\text { (c) } 2018 \text { The Author(s). Published by S. Karger AG, Basel } \\
\text { www.karger.com/cpb }\end{array}$
\end{tabular}

\section{Introduction}

In the United States, there are estimated more than 1.4 million men and women living with a diagnosis of colorectal cancer (CRC)[1]. With high recurrence rates and metastases, and nearly half of cases are diagnosed as late, CRC becomes the third leading cause of cancerrelated mortality both in men and women worldwide [2].

Hypoxia, or diminished availability of oxygen, is a common characteristic of solid tumors including CRC, in which proliferating tumor cells are oxygen deprived because of aberrant tumor microvasculature and inadequate blood supply. The hypoxic response is primarily attributed to hypoxia-inducible factor (HIF) in the tumor microenvironment [3, 4]. Hypoxiainducible factor $1 \alpha$ (HIF- $1 \alpha)$ is a heterodimeric transcription factor that is activated during hypoxic oxygen levels and regulates the expression of several genes [5-8] involved in cell metastasis and angiogenesis. Moreover, overexpression of HIF1a has been associated with a poor prognostic outcome in multiple types of cancer, including poor prognostic factors for CRC[9-12].

Serglycin (SRGN) encoded by the $S R G N$ is a hematopoietic cell granule proteoglycan, and aberrant expression of $S R G N$ have been associated with tumor progression and metastasis, in breast cancer [13], nasopharyngeal carcinoma [14] and non-small cell lung cancer [15]. SRGN is constitutively secreted from the cells and integrated into the extracellular matrix [16]. Studies have shown that SRGN is mainly expressed in all normal hematopoietic cells [17], endothelial cells [18, 19], tumor cells [20] and embryonic stem cells [21]. Moreover, serglycin plays an essential role in the formation of mast cell secretory granules and mediates the storage and secretion of various proteases, chemokines, and cytokines. Thus, SRGN is suggested to be highly correlated with CRC metastasis

Therefore, the present study aimed to investigate the expression and biological function of $S R G N$ in CRC cells and elucidated whether HIF- $1 \alpha$ could activate SRGN.

\section{Materials and Methods}

\section{Study Subjects}

This retrospective study included 105 patients with colorectal adenocarcinoma who received treatment at The Second Hospital of Tianjin Medical University between 2010 and 2015. This study was approved by The Tianjin Medical University Second Hospital Ethics Committee and written informed consent was obtained from each patient.

\section{Evaluation}

American Joint Committee on Cancer (AJCC) stages of the colorectal tumor lymph node was determined according to the seventh edition AJCC staging system using available clinical and pathologic tumor, node, and metastasis data. The same pathologist had evaluated both the diagnosis of the primary tumor resection and progression of the disease. Patient demographic characteristics including age, sex, clinicopathologic data such as tumor size, histological type, grade and TNM staging, the effect of treatment and clinical outcomes (overall survival) from hospital records were collected.

\section{Cell culture and hypoxia treatment}

Human CRC cell lines HT29, HCT8, and LS180 were obtained from the Chinese Academy of Sciences Type Culture Collection (Shanghai, China). All cells were cultured in DMEM/F12 or PRIM 1640 supplemented with $10 \%$ fetal bovine serum $(\mathrm{v} / \mathrm{v})$ in a humidified atmosphere of $5 \% \mathrm{CO}_{2}$ at $37{ }^{\circ} \mathrm{C}$. For hypoxic exposure treatment, the cells were placed in a modulator incubator (Thermoelectric company) that was flushed with a gas mixture consisting of $5 \% \mathrm{CO}_{2} 1.5 \% \mathrm{O}_{2}$ with balance $\mathrm{N}_{2}$, sealed, and incubated at $37^{\circ} \mathrm{C}$. 


\section{Cellular Physiology Cell Physiol Biochem 2018;48:2429-2440 \begin{tabular}{ll|l} 
and Biochemistry Published online: 15 August, 2018 & $\begin{array}{l}\text { (c) } 2018 \text { The Author(s). Published by S. Karger AG, Basel } \\
\text { www.karger.com/cpb }\end{array}$ \\
\hline
\end{tabular}}

\section{Establishment of stable cell lines}

A pCDH-puro lentiviral vector overexpressing CDS region of $H I F 1 \alpha$ and $S R G N$ was used. Moreover, for the knockdown experiment, a pLKO.1-bsd Lentiviral vector encoding shRNA SRGN to silence SRGN expression and a control vector with scrambled RNA was prepared by following the user manual. Lentivirus was produced in HEK293T cells by cotransfection with the control plasmid, expression plasmid or the packaging plasmid, using Lipofectamine 2000 (Invitrogen, 11668027) transfection reagent according to manufacturer's instructions. Culture medium containing lentiviral particles was collected $48 \mathrm{hr}$ post-transfection. Viral supernatants were pooled and stored at $-80^{\circ} \mathrm{C}$. This virus $(2 \mathrm{ml})$ was used to infect HT-29, HCT-8 and LS180 cells pretreated with Polybrene $(2 \mu \mathrm{L})$ at $37^{\circ} \mathrm{C}$ for $30 \mathrm{~min}$. Stable cells expressing HIF1 $\alpha$ and $S R G N$ were selected using $2 \mu \mathrm{g} / \mathrm{mL}$ puromycin and $4 \mu \mathrm{g} / \mathrm{mL}$ Bsd for 3 days.

\section{Western blot analysis}

Cells were harvested, washed and the total proteins were extracted in ice-cold RIPA buffer supplemented with proteases and phosphatases inhibitors (Sigma-Aldrich, Sigma, USA). The protein concentration was quantified using the Pierce Protein Assay Kit (Pierce, 23227). For western blot assay, an equal amount of protein was separated by electrophoresis on SDS-PAGE gel and transferred to polyvinylidene difluoride membranes (Merck Millipore, USA). Membranes were incubated with 5\% non-fat milk in TBS (Tris-buffered saline) buffer and probed with various primary antibodies HIF1 $\alpha$ (Abcam, ab113642, 1:1000); SRGN (Abcam, ab211515, 1:500); $\beta$-actin, GAPDH, $\beta$-Tubulin (Beijing Ray Antibody Biotech, RM2003, RM2002, RM2001, 1:5000). The membranes were washed three times with TBST and incubated with horseradish peroxidase (HRP)-conjugated secondary antibody (Cell Signaling Technology, anti-rabbit IgG 7074S or antimouse IgG 7076S, 1:5000). Specific antibody binding was detected using enhanced chemiluminescence detection reagents (Pierce).

\section{Quantitative Real-time PCR ( $q R T-P C R)$}

Total RNA was extracted from transfected cells using TRIzol Reagent (Invitrogen) by following the manufacturer's instructions (Invitrogen). cDNA was generated using SuperScriptIII cDNA kit (Invitrogen, 18080200). Reverse transcription PCR was performed using (RT-PCR, TaKaRa, RR014A) on an FTC-3000 ${ }^{\mathrm{TM}}$ System (Funglyn Biotech Inc., Toronto, Canada), using a first strand synthesis system. All experiments were performed in triplicate each sample was treated in triplicate and $\beta$-actin was used as an internal control. Each experiment was repeated at least three times independently. Relative quantitation was calculated using the $2^{-\Delta \Delta \mathrm{Ct}}$ method. The PCR primers used were listed in Table 1.

\section{Chromatin-Immunoprecipitation (ChIP) and Luciferase Assay}

Cells were cultured under normoxia or hypoxia for $12 \mathrm{hr}$, and then $1 \%$ formaldehyde was added to the cells to cross-link the chromatin proteins to DNA for $10 \mathrm{~min}$, and the reaction was stopped with $0.125 \mathrm{M}$ glycine for $5 \mathrm{~min}$. ChIP was performed according to manufacturer's instructions (Millipore, 17371). Cell lysates were sonicated and incubated with the specific antibodies overnight. HIF1 $\alpha$ Eluted DNA was analyzed by PCR and visualized on agarose gel electrophoresis. HCT-8 cells were seeded onto 6-well

Table 1. List of Primers used in the study

\begin{tabular}{|c|c|c|}
\hline HIF $1 \alpha$ for $\mathrm{qPCR}$ & Foward & TTCCCGACTAGGCCCATTC \\
\hline \multirow{3}{*}{ SRGN for qPCR } & Reverse & CAGGTATTCAAGGTCCCATTTCA \\
\hline & Foward & GGACTACTCTGGATCAGGCTT \\
\hline & Reverse & CAAGAGACCTAAGGTTGTCATGG \\
\hline \multirow[t]{2}{*}{ SRGN-HRE1 for ChIP } & Foward & ССССТTTCTTTCTGGGTTTT \\
\hline & Reverse & AGGGTGCTCTCTGTCССTTT \\
\hline \multirow[t]{2}{*}{ SRGN-HRE2 for ChIP } & Foward & TCTGGGTTTTGATGTGGATG \\
\hline & Reverse & GGAAATGTAGCAGGGTGCTC \\
\hline pGL3-SRGN-homo-MluI & Foward & CGACGCGTGGGTTGGTTACTGTGCTTGG \\
\hline pGL3-SRGN-homo-BglII & Reverse & GGAAGATCTAAGATGGCTGGGGAACAAGA \\
\hline SRGN-HRE1 MUT & Foward & TATTTGTTCAGGAAATTGTGTTTTTTGTTCTGGGCAGGGTTTGAG \\
\hline SRGN-HRE1 MUT & Reverse & TATTTGTTCAGGAAATTGTGTTTTTTGTTCTGGGCAGGGTTTGAG \\
\hline SRGN-HRE2 MUT & Foward & ATGAGAATGGAAAGTGGGGCTTTTTCATTAAGCCGTCTGGGGGAG \\
\hline SRGN-HRE2 MUT & Reverse & ATGAGAATGGAAAGTGGGGCTTTTTCATTAAGCCGTCTGGGGGAG \\
\hline
\end{tabular}




\section{Cellular Physiology Cell Physiol Biochem 2018;48:2429-2440 \begin{tabular}{l|l|l} 
DOI: 10.1159/000492657 & $\begin{array}{l}\text { O 2018 The Author(s). Published by S. Karger AG, Basel } \\
\text { www.karger.com/cpb }\end{array}$
\end{tabular}

plates at a $2 \times 10^{6}$ cells/well and were transfected with Lipofectamine 2000 reagent with plasmids pGL3 Basic-HRE-Promoter and its corresponding HRE mutant plasmid pGL3 Basic-HRE-mut and cultured in a medium under normoxia or hypoxia. GL2 luciferase plasmids were cotransfected with a pRL-null plasmid encoding Renilla luciferase (Promega). All transfections were carried out using FuGENE 6 transfection reagent (Roche), and luciferase activity was measured using the dual luciferase assay system (Promega). The cells were incubated for $48 \mathrm{hr}$ after transfection. Renilla activity was measured in cell lysates using the Dual-Luciferase Reporter Assay System (Promega, E1910) with a luminometer Filter Max F5 Multimode Microplate Reader. Results are expressed as fold change and represent the mean \pm SD of at least 3 experiments, each performed in triplicate.

\section{Wound healing and cell migration/invasion test}

For the cell scratch-wound assays, the transfected cells were grown to 80\% confluency in 6-well plates and were then incubated overnight. The cultured cells were then scratched to generate a wound in cell monolayer using sterilized pipette tip. The cells were then washed with PBS for 2-3 times. The cells were visualized under a light microscope at 24 and 48 hours. The cells in the wounded monolayer were photographed at different time points.

The migration was measured using an $8.0 \mu \mathrm{m}$ hole insert in 24-well transwell. For this assay, $1 \times 10^{5}$ cells in $200 \mu \mathrm{l}$ serum-free medium were seeded to the top chamber of transwell with DMEM/F12 or PRIM 1640. Trademarks coated with $1 / 4$ dilution Matrigel (Corning, 354234) were subjected to invasion assays. PRIM 1640 with 10\% FBS was added to the bottom chamber, and the cells were incubated for 8 or 24 hours. Cells that have migrated to the bottom of the filter were stained with $0.1 \%$ crystal violet (Thermo Scientific, 3300 ) for $30 \mathrm{~min}$ and counted under a microscope. All experiments were repeated at least three times independently.

\section{Immunohistochemistry (IHC)}

Immunohistochemical expression of HIF1 $\alpha$ and SRGN in tissue samples of patients with CRC was analyzed using the DAB Substrate Kit (Maxin, DAB-1031). Both the intensity and extent of immunological staining were analyzed semi-quantitatively. The staining intensity was scored with 0 used for negative staining, 1 for low or weakly positive, 2 for moderately positive and 3 for high or strongly positive. According to the percentage of positively stained cells, the sections were graded by four levels: 0 ( $0 \%$ staining), 1 (1\% $-25 \%), 2(26 \%-50 \%)$ and $3(51 \%-100 \%)$. The final score was determined by multiplying the intensity score with the extent of positivity scores of stained cells, with the minimum score of 0 and a maximum score of 9. A score of less than 2 is considered negative (-), 2-3 low expression $(+)$, 4-6 medium dyeing $(++),>6$ high staining expression $(+++)$. The score $\leq 5$ and $>5$ defined low and high, respectively.

\section{Statistical analysis}

Statistical analysis of clinical data was performed using the software package SPSS 17.0 software (IBM, Chicago, IL, USA). A two-tailed $\chi^{2}$ test or the Fisher exact test was used to determine the significance of differences among covariates (the clinicopathological data). All in vitro experiments were performed in triplicate and at least three times. Data were presented either as means \pm standard deviation (SD) from one representative independent experiment of three with similar results or means \pm standard error of the mean (SEM) from three independent experiments. Variables were analyzed by using both the Student t-test (twotailed) or one-way analysis of variance. A $P$ values less than 0.05 were considered statistically significant.

\section{Results}

Association of overexpression of SRGN with clinicopathological characteristics of CRC

We compared the protein and mRNA expression of SRGN in 10 CRC and paired adjacent normal tissues specimens. Despite interindividual variations, both SRGN protein (Fig. 1A, 1B) and mRNA (Fig. 1C) expressions were found to be significantly upregulated in CRC samples as compared with adjacent normal tissues, suggesting that SRGN was activated at transcriptional levels during CRC progression. Immunohistochemical protein expression analysis demonstrated that expression of SRGN was statistically significant 


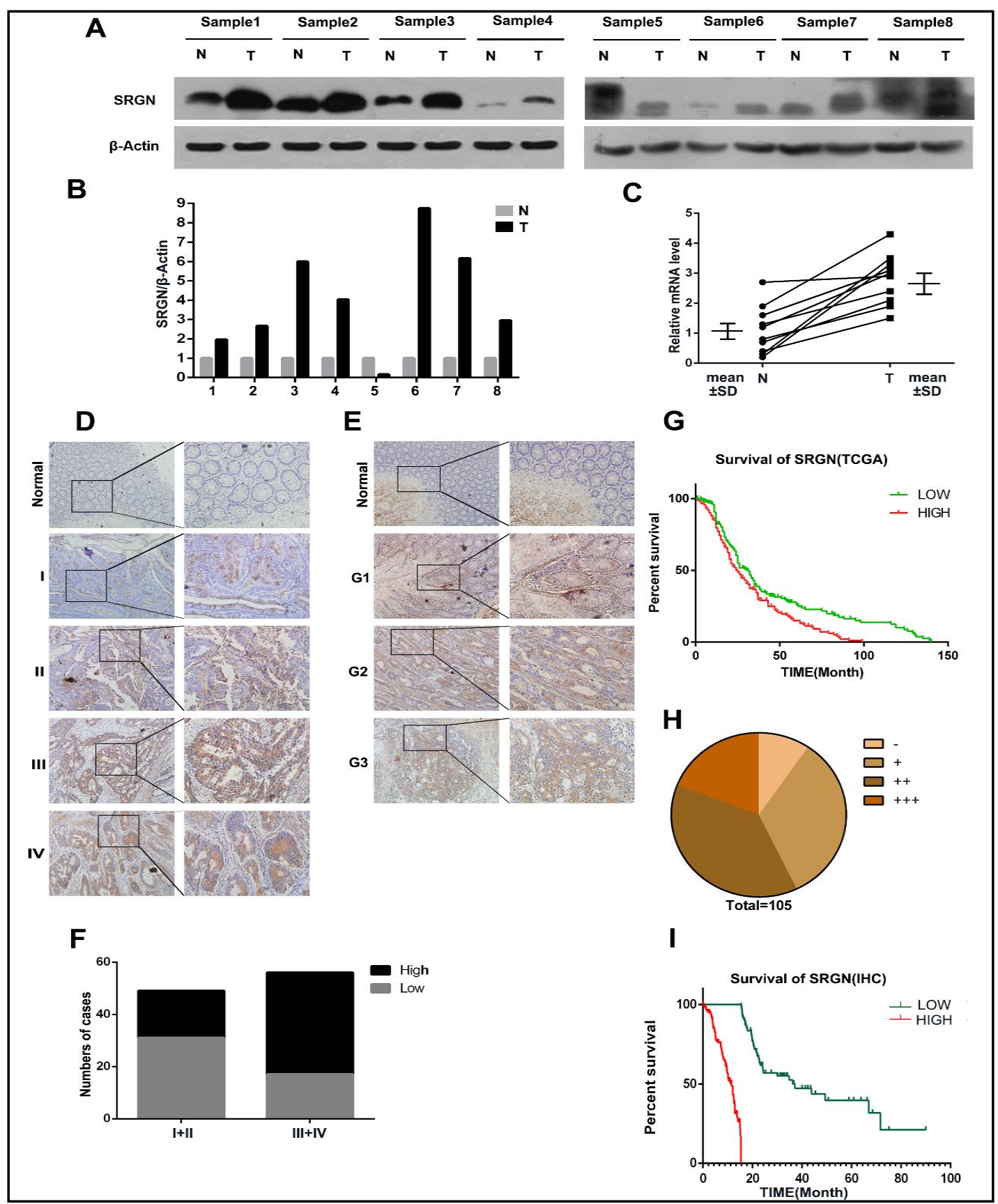

Fig. 1. SRGN is overexpressed in the CRC specimens. A. Western blot analysis of SRGN levels in paired human CRC tumors and matched adjacent normal tissues. B. SRGN protein expression levels were normalized to the expression of $\beta$-actin. C. Quantitative RT-PCR (qRT-PCR) analysis of SRGN expression inpaired human CRC tumors and matched adjacent normal tissues $\left({ }^{*} \mathrm{P}<0.01\right)$. D. Representative images of SRGN protein in different stages of CRC by IHC. E. Expression analysis of SRGN protein among different grades of CRC by IHC. F. Association of overexpression of SRGN with metastasis differentiation stages in CRCs. G. Association between SRGN mRNA expression levels and the overall survival of patients with CRC (data from TCGA). H. The distribution of SRGN protein expression in patients with CRC. I. The overall survival of patients with CRC based on IHC staining intensity (The score $\leq 5$, Low; $>5$, high). N, normal; $\mathrm{T}$, tumor. 
among different stages (Fig. 1D); however, there was no significant difference among different grades (Fig. 1E). High positive staining of SRGN was observed in metastasis cases of CRC compared with non-metastasis cases (Fig. 1F). Furthermore, we have analyzed data from The Cancer Genome Atlas (TCGA) and found that CRC patients with high mRNA expression of SRGN had shorter OS compare to those with low expression of $\operatorname{SRGN}(P<0.001)$ (Fig. $1 G)$. Similar findings were observed when we analyzed and compared the expression of SRGN in 105 primary CRC specimens (Fig. 1H, 1I). Further to determine the role of SRGN in the pathogenesis of CRC, we evaluated the association of SRGN protein expression in CRC with clinicopathological characteristics. However, there was no significant association between SRGN expression and age, gender or histological differentiation $(P>0.05)$ (Table 2). These findings indicated that SRGN may play critical roles in CRC development and progression and may serve as a potentially valuable biomarker for CRC.

\section{HIF-1 $\alpha$ regulated the overexpression of SRGN under hypoxic conditions in CRC cells}

To determine whether SRGN expression could be induced under the microenvironment hypoxia, a hallmark of solid tumors including CRC, we exposed HT29, HCT8, and LS180 cells to hypoxic conditions $\left(1.5 \% \mathrm{O}_{2}\right)$ for 12 hours. Noticeably, we found that the expression of SRGN in CRC cells both at the mRNA and protein levels increased significantly under hypoxic conditions, moreover, HIF-1 $\alpha$ protein expression also increased rapidly in these cell lines (Fig. 2A and 2B). Compared to the HT29, HCT8 and LS180 cells with basal level expression of HIF-1 $\alpha$ (Fig. 2C), HT29 and HCT8 cells exhibiting overexpression of HIF-1 $\alpha$ markedly upregulated the expression of SRGN both at the mRNA (Fig. 2D) and protein (Fig. $2 \mathrm{E})$ levels under normoxia; however, HIF- $1 \alpha$ knockdown remarkably reduced the expression of SRGN in HCT8 and LS180 cells (Fig. 2F and 2G) with normoxia. These results suggested that hypoxia induces overexpression of SRGN in CRC cells and HIF-1 $\alpha$ is involved in this regulatory mechanism.

Next, we also analyzed the mRNA expression data from TCGA, on SRGN and HIF-1 $\alpha$ and found a significant positive association between the two (Fig. $2 \mathrm{H}$ ). On further stratification of HIF- $1 \alpha$ mRNA into low and high groups based on the median level of expression, SRGN mRNA level showed statistical significance (Fig. 2I). We performed IHC analysis of HIF- $1 \alpha$ and SRGN expression in tissue specimens obtained from the same patient with CRC. As shown in Fig. 2J, SRGN protein colocalized with HIF1 $\alpha$ in consecutive sections of CRC tissue specimen. Furthermore, statistical analysis revealed that the protein expression of SRGN significantly positively correlated with that of HIF- $1 \alpha$ in the CRC specimens $\left(\chi^{2}=24.681 ; P<0.001\right)$ (Fig. $2 \mathrm{~K})$. 


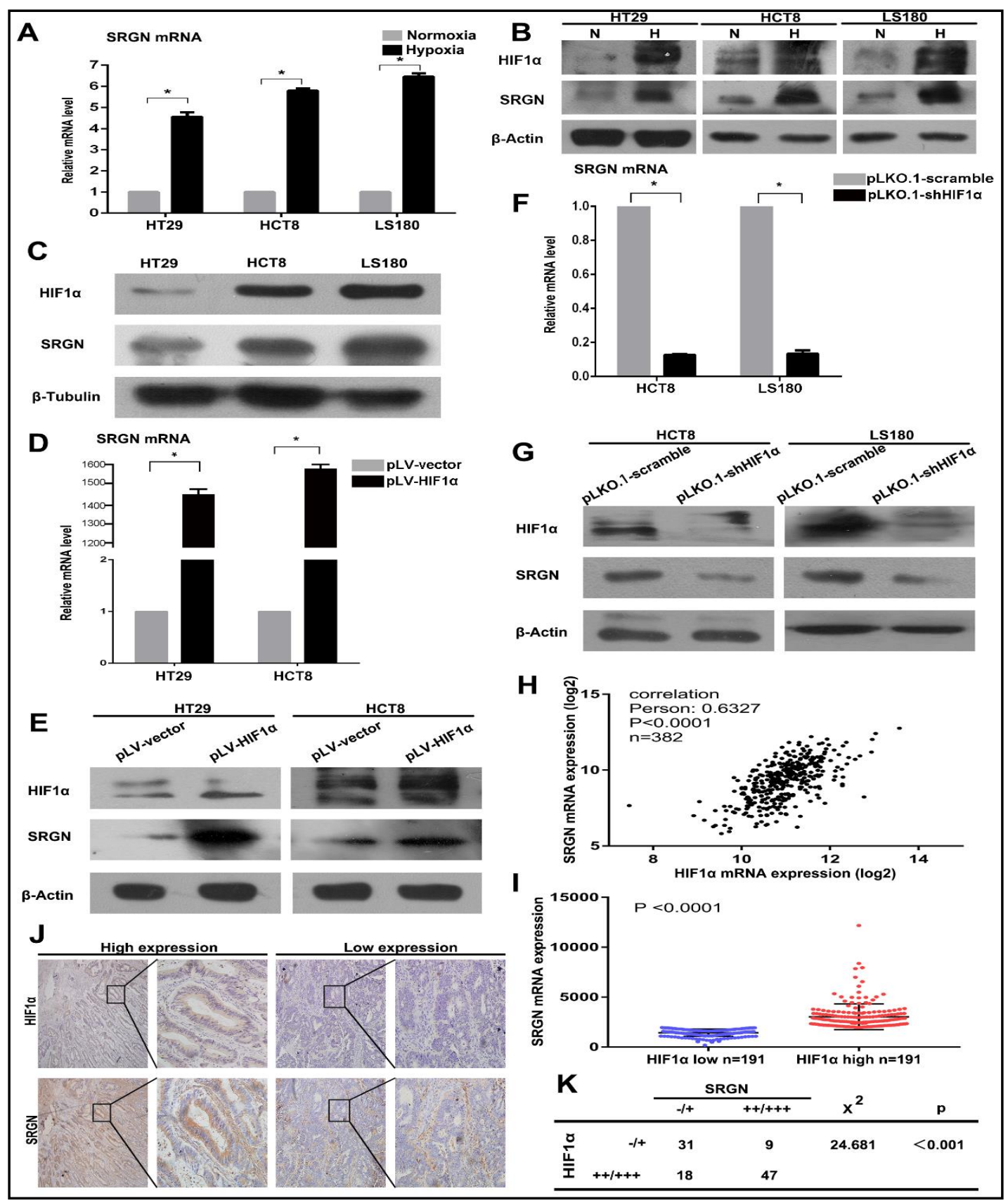

Fig. 2. SRGN is a novel HIF1 $\alpha$ target gene. A. SRGN mRNA level by qRT-PCR in three human CRC cell lines under normoxia $\left(21 \% \mathrm{O}_{2}\right)$ and hypoxia $\left(1.5 \% \mathrm{O}_{2}\right)$ for 12 hours. B. HIF1 $\alpha$ and SRGN expression was determined by Western blot analysis in three human CRC cell lines cultured under normoxia and hypoxia conditions. C. Western blot analysis of HIF1 $\alpha$ and SRGN protein expression in 3 human CRC cell lines. D. SRGN mRNA expression by qRT-PCR in HT29 and HCT8 pLV-vector or pLV-HIF1 $\alpha$ stable cell lines. E. Western blot of HIF1 $\alpha$ and SRGN expression in pLV-HIF1 $\alpha$ stable cell lines compared with control stable cell lines. F. SRGN mRNA expression by qRT-PCR in HCT8 and LS180 pLKO.1-scramble or pLKO.1-shHIF1 $\alpha$ stable cell lines. G. Western blot of HIF1 $\alpha$ and SRGN expression in pLKO.1-shHIF1 $\alpha$ stable cell lines compared with control stable cell lines. H. Pearson correlation analyses for the association of SRGN with HIF-1 $\alpha$ mRNA expression (data from TCGA). I. SRGN mRNA level between the two groups of HIF-1 $\alpha$ mRNA low and high (data from TCGA). J. Colocalization of SRGN and HIF-1 $\alpha$ in consecutive sections of CRC specimens by IHC staining. K. Statistical analysis of IHC results of HIF-1 $\alpha$ and SRGN expression in human CRC surgical samples. $\mathrm{N}$, normoxia; $\mathrm{H}$, hypoxia. 
Association between HIF$1 \alpha$ and SRGN expression in CRC cells

Based on western blot analysis of SRGN and HIF1 $\alpha$ expression in 3 CRC cell lines (Fig. 2C), we constructed pLVSRGN or pLKO.1-shSRGN and controlled stable cell lines and confirmed the expression at mRNA (Fig.3A, 3B) and protein (Fig.3C, 3D) level. To explicit whether HIF1 $\alpha$ induces $S R G N$, we used the blocking and rescue experiment using western blot (Fig. 3E).

Next, to further understand the molecular mechanism underlying SRGN overexpression in CRC under hypoxia, we performed a bioinformatic analysis to predict the potential putative binding sites in the promoter region of human SRGN gene and identified two hypoxia response elements (HREs) (Fig. 3F, up). To investigate whether HIF1a physically binds to the HREs of SRGN promoter, and transactivate its expression, chromatin immunoprecipitation (CHIP) assay was performed in HCT8 cells at $1.5 \% \mathrm{O}_{2}$ or $21 \% \mathrm{O}_{2}$. The PCR analysis revealed that only the HRE of SRGN promoter located between 1, 713 to 1,708 was detected (Fig. 3F, bottom), suggesting that hypoxia promoted the binding of HIF $1 \alpha$ to SRGN promoter. Moreover, to determine whether the HRE2 site is also required for HIF $1 \alpha$ to transactivate $S R G N$ promoter, the consensus HIF1 $\alpha$ sequence was mutated from ACGTG to ACATG. As shown in Fig. 3G, the mutation in HRE2 nearly abolished the transactivation of SRGN promoter by HIF $1 \alpha$.

\section{SRGN overexpression promoted cell migration and invasion in CRC}

An in vitro scratch assay and transwell assays were used understand the role of SRGN in HIF- $1 \alpha$ mediated CRC cell migration; we overexpressed SRGN in HIF- $1 \alpha$ knockdown HCT8 and LS180 cells. The cells were wounded by scratching and cultured them for 24-48 hours. Results showed that the migratory activity of HT29 and HCT8 was also inhibited by SRGN

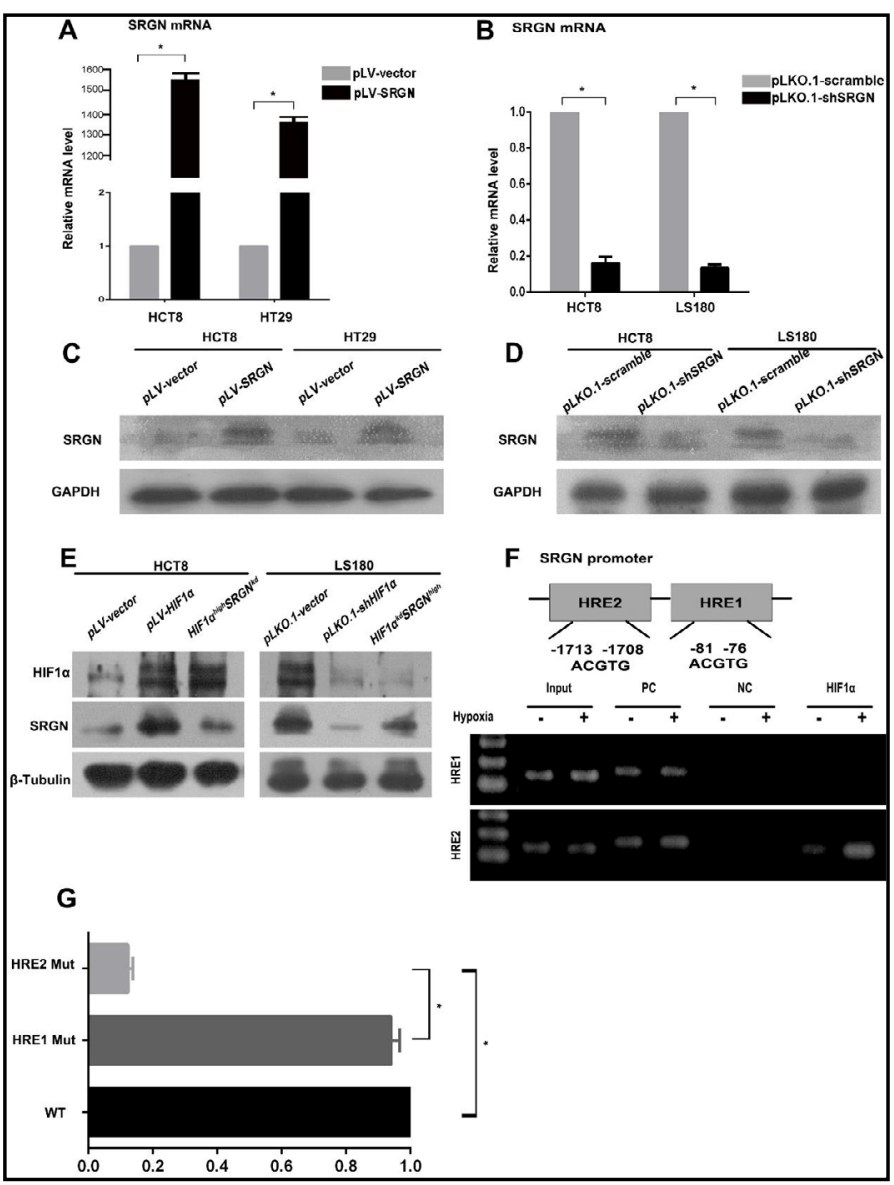

Fig. 3. Correlation of SRGN with HIF-1 $\alpha$. A. B. SRGN mRNA expression in HT29 and HCT8 pLV-vector or pLV-SRGN (left), HCT8 and LS180 pLKO.1-scramble or pLKO.1-shSRGN (right) stable cell lines. C. D. SRGN protein expression by Western blot in HT29 and HCT8 pLV-vector or pLV-SRGN stable cell lines(left) and HCT8 and LS180 pLKO.1-scramble or pLKO.1-shSRGN stable cell lines(right). E. HCT8 (left) and LS180 (right) cells were infected with pLV-HIF1 $\alpha$, HIF $1 \alpha^{\text {high }}$ SRGN $^{\mathrm{kd}}$ or pLKO.1-shHIF $1 \alpha$, HIF $1 \alpha^{\text {kd }}$ SRGN ${ }^{\text {high }}$ respectively assessed by Western blot analysis. F. Two HREs located in SRGN promoter at the different sites (top); chromatin immunoprecipitation (CHIP) analysis of HIF1 $\alpha$ binding to SRGN promoter in HCT8 cells (bottom). G. Luciferase reporter assay results demonstrated that deletion of the region from -1713 to $-1708 \mathrm{bp}$, encompassing the HRE2 site, markedly reduced the promoter activity of SRGN induced by HIF-1 $\alpha$. 
Fig. 4. SRGN Increased CRC cell mobility with normoxia. A.B. Wound-healing assays comparing the motility of HCT8 (left) and HT29 (right) with pLV-vector, pLV-HIF1 $\alpha$ or HIF1 $\alpha^{\text {high }}$ SRGN ${ }^{k d}$ stable cell lines, respectively for 24-48 hours, quantification of the relative migration distance of HCT8 (left) and HT29 (right). C.D. Wound-healing assays comparing the motility of НСТ8 (left) and LS180 (right) with pLKO.1scramble, pLKO.1-shHIF1 $\alpha$ or HIF1 $\alpha^{\text {kd }}$ SRGN ${ }^{\text {high }}$ stable cell lines, respectively for 24-48 hours, quantification of the relative migration distance of HCT8 (left) and LS180 (right). E.F. Comparison of migration and invasion potential of HCT8 (left) and HT29 (right) cells transfected with pLV-vector, pLV-HIF $1 \alpha$ or HIF1 $\alpha^{\text {high }}$ SRGN ${ }^{k d}$ for 8-12 hours using Boyden chambers, number of cells in migration and invasion test of HCT8 (left) and HT29 (right). G.H. Comparison of migration and invasion potential of HCT8 (left) and HT29 (right) cells transfected with pLV-vector, pLV-HIF1 $\alpha$ or HIF1 $\alpha^{\text {high }} S R G N^{k d}$ for

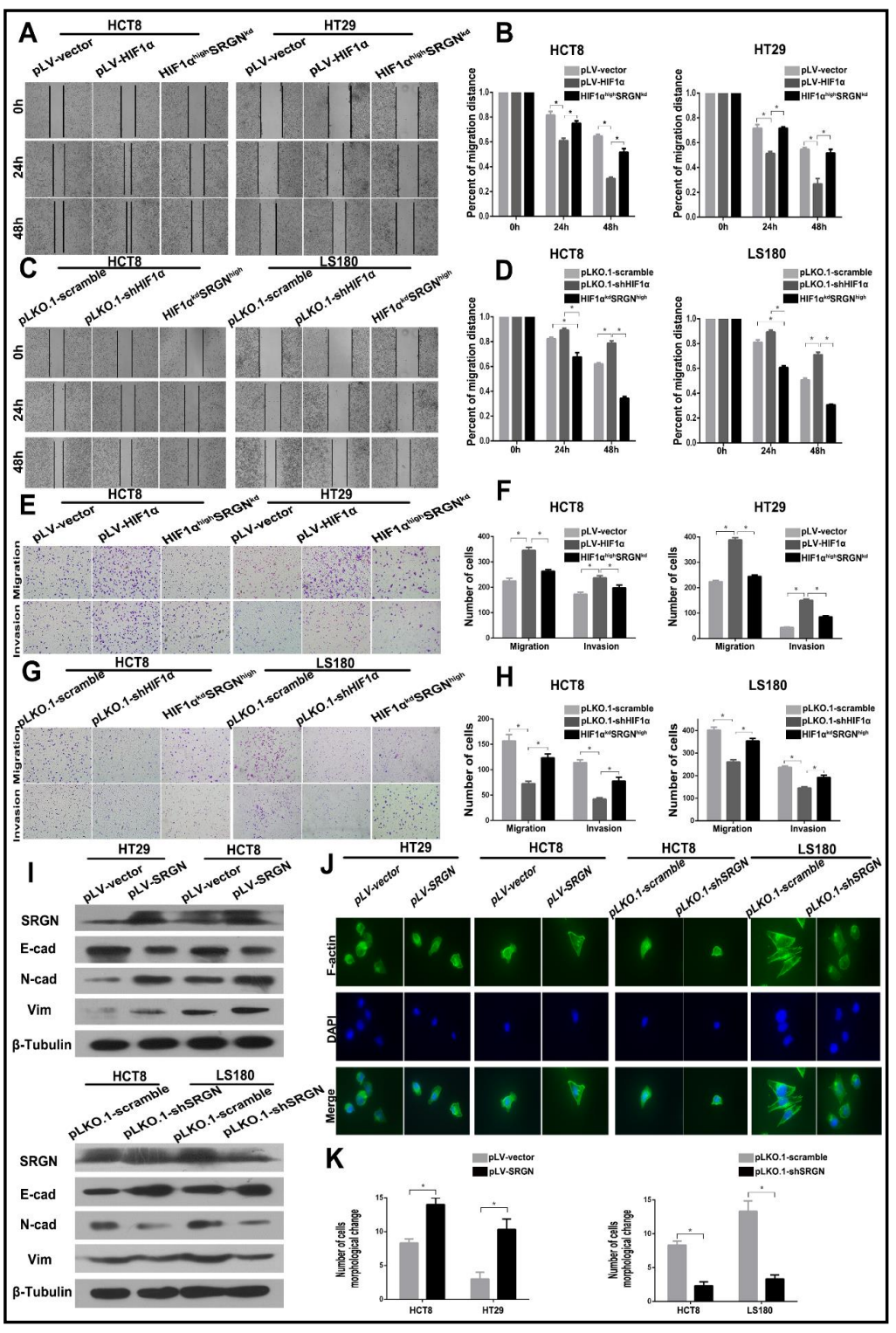
8-12 hours using Boyden chambers, number of cells in migration and invasion test of HCT8 (left) and HT29 (right). I. Western blots analysis of EMT related markers in HT29 and HCT8 (up) pLV-vector or pLV-SRGN stable cells, in HCT8 and LS80 (bottom) pLKO.1-scramble or pLK0.1.1-shSRGN stable cells. J. F-actin (green) and DAPI (blue) for HT29 and HCT8 (left) pLV-vector or pLV-SRGN stable stable cells, for HCT8 and LS80 (right) pLKO.1scramble or pLKO.1-shSRGN stable cells by Immunofluorescence staining. K. The number of morphological change for HT29 and HCT8 (left) pLV-vector or pLV-SRGN stable stable cells, for HCT8 and LS80 (right) pLKO.1-scramble or pLKO.1-shSRGN stable cells.

silencing in HIF-1 $\alpha$ overexpressing cells (Fig. 4A, 4B). Nevertheless, SRGN overexpression at least partially rescued the inhibitory effect of HIF- $1 \alpha$ knockdown on HCT8 and LS180 cell migration (Fig. 4C, 4D), suggesting that $S R G N$ was involved in HIF- $1 \alpha$ mediated invasion and migration.

Cell invasion assays using Boyden chamber confirmed these findings. Both the migratory ability and invasiveness of pLKO.1-shSRGN and pLV-HIF- $1 \alpha$ HCT8 and HT29 stable cells were markedly attenuated (Fig. 4E, 4F), whereas the migratory ability and invasiveness of SRGN in HIF-1 $\alpha$ knockdown HCT8 and LS180 cells were much higher than those of control cells 


\section{Cellular Physiology Cell Physiol Biochem 2018;48:2429-2440

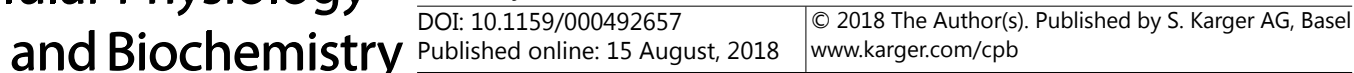 \\ Xu et al.: SRGN Promotes CRC Metastasis via EMT under Hypoxia}

(Fig. 4G, 4H). This findings further confirmed the oncogenic role of $S R G N$ in progression and metastasis of CRC. Taken together, these results indicated that overexpression of SRGN was sufficient to promote CRC cell migration and invasion.

To explore the association of SRGN with epithelial-mesenchymal transition (EMT), the protein levels of several EMT markers were evaluated. A high level of SRGN accompanied elevated expression of mesenchymal markers vimentin and $\mathrm{N}$-cadherin, a reduced level of epithelial protein E-cadherin in the HT29 and HCT8 pLV-SRGN stable cells; however, the suppression of $S R G N$ elicited lower vimentin and N-cadherin expression level, but a higher level of E-cadherin (Fig. 4I). Together, these results suggested that SRGN induces EMT.

The morphological alteration was observed by confocal microscopy via F-actin staining. Immunofluorescence analysis F-actin confirmed that HT29 and HCT8 pLV-SRGN exhibited some pseudopods or lamellipodia extending from cell bodies; interestingly, the morphology of HCT8 and LS180 pLKO.1-shSRGN cells lacked thin and long pseudopods (Fig. 4J, K). These data indicated that SRGN was critical for the migration and invasion of CRC cells.

\section{Discussion}

The present study investigated the role of SRGN in CRC metastasis. Our data demonstrated that SRGN expression levels were higher in CRC than in adjacent normal tissues. Our mechanistic study revealed that SRGN physically binds to an HRE on SRGN promoter and that overexpression in CRC was predominantly mediated by HIF $1 \alpha$. A positive correlation between SRGN expression and clinicopathologic features of patients with CRC was observed; however, a negative correlation between SRGN expression and overall survival (OS) was also revealed. Some studies have shown that SRGN promotes cell migration by EMT. Together, this study demonstrated that SRGN had a potent oncogenic role in promoting CRC migration and invasion. Considering these findings, we concluded that SRGN is a direct target of HIF-1 $\alpha$ in CRCs and play crucial roles in CRC progression.

Recent studies have revealed elevated expression of SRGN in a variety of cancers, including hepatocellular carcinoma [3], non-small cell lung cancer [15], and nasopharyngeal squamous cell carcinoma [14]. SRGN is a small molecule glycoprotein predominantly distributed in the cell cytoplasm and cell membrane; however, it can also be secreted and integrated into the extracellular matrix [22]. SRGN can be derived and utilized in exosomes to promote tumor growth and metastasis in multiple myeloma [23] and lung adenocarcinoma [24]; however, its role in CRC exosomes remains to be elucidated. Furthermore, considering a possible association between SRGN expression and its invasive potential, we suggest that SRGN may have severe impacts on mesenchymal cells or the surrounding tumor cells and necessitate further studies. In this context, we used F-actin as a distinctive of cell motor ability and found that elevated expression of SRGN significantly enhanced CRC migration and invasion, whereas SRGN knock-down significantly reduced the migration and invasion of CRC cells. All these findings are consistent with those reported previously, which strongly supported that SRGN may function as an oncogene in CRCs.

Hypoxic regions are a common characteristic of the microenvironment of the majority of solid tumors [25]. HIF-1 is a heterodimer comprised of HIF- $1 \alpha$ and HIF1 $\beta$ subunits. HIF- $1 \alpha$ functions as a master regulator of cellular and systemic homeostatic response to hypoxia [26-29]. Studies have found that in a sustained hypoxic environment, HIF-1 $\alpha$ was stabilized and prevented from undergoing protein degradation in a ubiquitin/proteasome pathway. HIF-1 $\alpha$ has long been known to be a transcriptional factor, inducing the expression of multiple target genes in favor of the tolerance of tumor cells to hypoxia, by recognizing HREs (5'-RCGTG-3'). Our results indicated that elevated HIF1 $\alpha$ levels in CRC transactivate SRGN gene transcription and protein expression. By upregulating SRGN, HIF1 $\alpha$ promotes the formation of membrane protrusions such as lamellipodia and filopodia. Taken together, these results indicated that HIF1 $\alpha /$ SRGN axle regulate CRC cells invasion and metastasis. 


\section{Cellular Physiology Cell Physiol Biochem 2018;48:2429-2440 \begin{tabular}{ll|l} 
and BiOChemistry & $\begin{array}{l}\text { DOI: 10.1159/000492657 } \\
\text { Published online: 15 August, } 2018\end{array}$ & $\begin{array}{l}\text { ( } 2018 \text { The Author(s). Published by S. Karger AG, Basel } \\
\text { www.karger.com/cpb }\end{array}$ \\
\hline
\end{tabular}

\section{Conclusion}

The present study provides both clinical and experimental evidence of $S R G N$ is an important oncogene in CRCs and that SRGN overexpression was significantly associated with CRC metastasis. In vitro SRGN overexpression promoted CRC migration and invasion. Mechanistically, the expression of SRGN in CRCs was transcriptionally activated by HIF$1 \alpha$, as an essential regulator of hypoxic microenvironments. Therefore, inhibition of SRGN expression may be more effective for treating metastatic CRC. In conclusion, we demonstrated that dysregulated HIF-1 $\alpha /$ SRGN signaling promotes CRC progression and metastasis. SRGN may serve as a potential therapeutic target for metastatic CRC.

\section{Acknowledgements}

Funding/Support: This work was supported by grants from National Natural Science Foundation of China (Grant No. 81472183) and The Second Hospital of Tianjin Medical University (Grant No. 2016dyey07).

\section{Disclosure Statement}

All authors declare that they have no conflicts of interest.

\section{References}

-1 Miller KD, Siegel RL, Lin CC, Mariotto AB, Kramer JL, Rowland JH, Stein KD, Alteri R, Jemal A: Cancer treatment and survivorship statistics, 2016. CA Cancer J Clin 2016;66:271-289.

-2 Vedeld HM, Goel A, Lind GE: Epigenetic biomarkers in gastrointestinal cancers: The current state and clinical perspectives. Semin Cancer Biol 2017; DOI:10.1016/j.semcancer.2017.12.004.

-3 Parks SK, Chiche J, Pouyssegur J: Disrupting proton dynamics and energy metabolism for cancer therapy. Nat Rev Cancer 2013;13:611-623.

-4 He C, Wang L, Zhang J, Xu H: Hypoxia-inducible microRNA-224 promotes the cell growth, migration and invasion by directly targeting RASSF8 in gastric cancer. Mol Cancer 2017;16:35.

5 Zhao T, Ren H, Li J, Chen J, Zhang H, Xin W, Sun Y, Sun L, Yang Y, Sun J, Wang X, Gao S, Huang C, Zhang H, Yang S, Hao J: LASP1 is a HIF1alpha target gene critical for metastasis of pancreatic cancer. Cancer Res 2015;75:111-119.

6 Kong F, Kong X, Du Y, Chen Y, Deng X, Zhu J, Du J, Li L, Jia Z, Xie D, Li Z, Xie K: STK33 Promotes Growth and Progression of Pancreatic Cancer as a Critical Downstream Mediator of HIF1alpha. Cancer Res 2017;77:6851-6862.

-7 Ren H, Jia L, Zhao T, Zhang H, Chen J, Yang S, Liu J, Yu M, Hao J: Hypoxia inducible factor (HIF)-1alpha directly activates leptin receptor (Ob-R) in pancreatic cancer cells. Cancer Lett 2014;354:172-180.

-8 Zhao X, Gao S, Ren H, Sun W, Zhang H, Sun J, Yang S, Hao J: Hypoxia-inducible factor-1 promotes pancreatic ductal adenocarcinoma invasion and metastasis by activating transcription of the actin-bundling protein fascin. Cancer Res 2014;74:2455-2464.

-9 Xia Y, Choi HK, Lee K: Recent advances in hypoxia-inducible factor (HIF)-1 inhibitors. Eur J Med Chem 2012;49:24-40.

10 Wilson WR, Hay MP: Targeting hypoxia in cancer therapy. Nat Rev Cancer 2011;11:393-410.

11 Sui H, Zhao J, Zhou L, Wen H, Deng W, Li C, Ji Q, Liu X, Feng Y, Chai N, Zhang Q, Cai J, Li Q: Tanshinone IIA inhibits beta-catenin/VEGF-mediated angiogenesis by targeting TGF-beta1 in normoxic and HIF-1alpha in hypoxic microenvironments in human colorectal cancer. Cancer Lett 2017;403:86-97.

12 Xiang J, Sun H, Su L, Liu L, Shan J, Shen J, Yang Z, Chen J, Zhong X, Avila MA, Yan X, Liu C, Qian C: Myocyte enhancer factor 2D promotes colorectal cancer angiogenesis downstream of hypoxia-inducible factor $1 \alpha$. Cancer Lett 2017;400:117-126. 


\section{Cellular Physiology Cell Physiol Biochem 2018;48:2429-2440 \begin{tabular}{ll|l} 
DOI: 10.1159/000492657 & $\begin{array}{l}\text { O } 2018 \text { The Author(s). Published by S. Karger AG, Basel } \\
\text { www.karger.com/cpb }\end{array}$
\end{tabular} \\ Xu et al.: SRGN Promotes CRC Metastasis via EMT under Hypoxia}

13 Zhang Z, Deng Y, Zheng G, Jia X, Xiong Y, Luo K, Qiu Q, Qiu N, Yin J, Lu M, Liu H, Gu Y, He Z: SRGN-TGFbeta2 regulatory loop confers invasion and metastasis in triple-negative breast cancer. Oncogenesis 2017;6:e360.

14 Li XJ, Ong CK, Cao Y, Xiang YQ, Shao JY, Ooi A, Peng LX, Lu WH, Zhang Z, Petillo D, Qin L, Bao YN, Zheng FJ, Chia CS, Iyer NG, Kang TB, Zeng YX, Soo KC, Trent JM, Teh BT, et al.: Serglycin is a theranostic target in nasopharyngeal carcinoma that promotes metastasis. Cancer Res 2011;71:3162-3172.

15 Guo JY, Hsu HS, Tyan SW, Li FY, Shew JY, Lee WH, Chen JY: Serglycin in tumor microenvironment promotes non-small cell lung cancer aggressiveness in a CD44-dependent manner. Oncogene 2017;36:2457-2471.

16 Kolset SO, Zernichow L: Serglycin and secretion in human monocytes. Glycoconj J 2008;25:305-311.

17 Pejler G, Abrink M, Wernersson S: Serglycin proteoglycan: regulating the storage and activities of hematopoietic proteases. Biofactors 2009;35:61-68.

18 Woulfe DS, Lilliendahl JK, August S, Rauova L, Kowalska MA, Abrink M, Pejler G, White JG, Schick BP: Serglycin proteoglycan deletion induces defects in platelet aggregation and thrombus formation in mice. Blood 2008;111:3458-3467.

19 Schick BP, Gradowski JF, San Antonio JD: Synthesis, secretion, and subcellular localization of serglycin proteoglycan in human endothelial cells. Blood 2001;97:449-458.

20 Baghy K, Tatrai P, Regos E, Kovalszky I: Proteoglycans in liver cancer. World J Gastroenterol 2016;22:379393.

21 Schick BP, Ho HC, Brodbeck KC, Wrigley CW, Klimas J: Serglycin proteoglycan expression and synthesis in embryonic stem cells. Biochim Biophys Acta 2003;1593:259-267.

-22 Schick BP: Serglycin proteoglycan deletion in mouse platelets: physiological effects and their implications for platelet contributions to thrombosis, inflammation, atherosclerosis, and metastasis. Prog Mol Biol Transl Sci 2010;93:235-287.

-23 Purushothaman A, Bandari SK, Chandrashekar DS, Jones RJ, Lee HC, Weber DM, Orlowski RZ: Chondroitin sulfate proteoglycan serglycin influences protein cargo loading and functions of tumor-derived exosomes. Oncotarget 2017;8:73723-73732.

24 Vykoukal J, Sun N, Aguilar-Bonavides C, Katayama H, Tanaka I, Fahrmann JF, Capello M, Fujimoto J, Aguilar M, Wistuba, II, Taguchi A, Ostrin EJ, Hanash SM: Plasma-derived extracellular vesicle proteins as a source of biomarkers for lung adenocarcinoma. Oncotarget 2017;8:95466-95480.

-25 Yang Y, Sun M, Wang L, Jiao B: HIFs, angiogenesis, and cancer. J Cell Biochem 2013;114:967-974.

26 Logsdon DP, Grimard M, Luo M, Shahda S, Jiang Y, Tong Y, Yu Z, Zyromski N, Schipani E, Carta F, Supuran CT, Korc M, Ivan M, Kelley MR, Fishel ML: Regulation of HIF1alpha under Hypoxia by APE1/Ref-1 Impacts CA9 Expression: Dual Targeting in Patient-Derived 3D Pancreatic Cancer Models. Mol Cancer Ther 2016;15:2722-2732.

27 Huang LE: Biochemistry. How HIF-1alpha handles stress. Science 2013;339:1285-1286.

28 Yoo YG, Christensen J, Huang LE: HIF-1alpha confers aggressive malignant traits on human tumor cells independent of its canonical transcriptional function. Cancer Res 2011;71:1244-1252.

29 Zhang C, Samanta D, Lu H, Bullen JW, Zhang H, Chen I, He X, Semenza GL: Hypoxia induces the breast cancer stem cell phenotype by HIF-dependent and ALKBH5-mediated m(6)A-demethylation of NANOG mRNA. Proc Natl Acad Sci U S A 2016;113:E2047-2056. 